\title{
Mobility dependent efficiencies of organic bulk heterojunction solar cells: Surface recombination and charge transfer state distribution
}

\author{
Thomas Kirchartz, ${ }^{1}$ Bart E. Pieters, ${ }^{1}$ Kurt Taretto, ${ }^{2}$ and Uwe Rau ${ }^{1}$ \\ ${ }^{1}$ IEF5-Photovoltaik, Forschungszentrum Jülich, 52425 Jülich, Germany \\ ${ }^{2}$ Departamento de Electrotecnia, Universidad Nacional del Comahue, Buenos Aires 1400, 8300 Neuquen, Argentina
}

(Received 7 May 2009; revised manuscript received 3 July 2009; published 31 July 2009)

\begin{abstract}
Recent simulations of the efficiency of polymer/fullerene solar cells as a function of mobility predicted finite optimum mobilities due to a decrease in open circuit voltage for higher mobilities. We explain this decrease in open circuit voltage with two features of the commonly used model, namely, infinite surface recombination and an integration over a distribution of separation distances of electron and hole in a charge transfer state at the interface between donor and acceptor molecules. Especially, the assumption of a variable electron/hole pair separation at the interface has a considerable influence on the open circuit voltage.
\end{abstract}

DOI: 10.1103/PhysRevB.80.035334

PACS number(s): 84.60.Jt, 72.80.Le

\section{INTRODUCTION}

The currently most efficient class of polymer-based solar cells has an absorber consisting of a blend of donor and acceptor molecules, which creates a heterojunction throughout the bulk of the device. ${ }^{1-4}$ Simulation of these so-called bulk heterojunction solar cells has to include several features specific to this type of solar cell. In contrast to most inorganic solar cells, charge separation takes place as a multistep process ${ }^{5}$ starting with (i) photon absorption and exciton generation. This exciton then (ii) has to diffuse to the interface between the donor and acceptor molecules where it is separated. A frequently used model ${ }^{6-11}$ for the simulation of current/voltage curves of organic bulk heterojunction solar cells assumes that the dissociated exciton (iii) first creates a charge transfer state ${ }^{12,13}$ at the interface, before (iv) the electron and hole are separated and (v) transported to their respective junctions. A feature very uncommon to the classical picture of inorganic solar cells results from the interplay between electric field dependent dissociation and nonradiative recombination of the charge transfer state as outlined in Ref. 8 . This model predicts a strong decrease in open circuit voltage with increasing charge-carrier mobility leading eventually to a maximum of the device efficiency of organic bulk heterojunction solar cells at a finite optimum mobility. ${ }^{14,15}$ The possibility that carrier mobilities in organic bulk heterojunction solar cells beyond the optimum mobility reduces the device efficiency, or more generally speaking, that faster kinetics leads to lower performance, appears counterintuitive, and would be a phenomenon that is different from mobility effects in classical inorganic ( $p n$-junction) solar cells. ${ }^{16}$

This paper aims at an improved understanding of the reasons for a finite optimum mobility in a device model that includes a charge transfer state. We show that such an optimum can originate from two rather different features of the model that have not been explicitly named until now. The first feature is a high (or even infinite) surface recombination that is usually implicitly assumed in organic solar-cell modeling. Such an assumption is, in principle, also possible in inorganic solar cells with insufficient passivation of the surfaces. However, passivation of surfaces for decreased contact recombination is a general challenge for every solar-cell technology and is therefore, no specific property of organic photovoltaics. The second feature originates from the assumption of a distribution of separation distances of the electron and the hole in the charge transfer state. ${ }^{17}$ We will show that this apparently minor variation in the original OnsagerBraun theory ${ }^{6,18}$ has a severe influence on open circuit voltage and its evolution versus mobility. Thus, this contribution wants to focus attention on the question in how far the assumption of charge transfer state distributions is physically valid and necessary for organic solar-cell modeling.

\section{THEORY}

Organic solar-cell efficiencies may decrease for higher mobilities, when simulating the current/voltage curve using a one-dimensional drift-diffusion model combined with Onsager-Braun theory. ${ }^{14,15}$ The key for a better understanding and critical evaluation of the reasons for this counterintuitive result is the principle of detailed balance. ${ }^{19-22}$ Figure 1(a) shows a simplified scheme of the interaction between the charge transfer state and the free electrons and holes. The concentration $\xi$ of charge transfer excitons depends on the coupling constant $k_{\text {rec }}$ describing the interaction of charge transfer excitons with the free carriers, the recombination constant $k_{\mathrm{f}}$ and the generation rate $G$. This generation rate implicitly includes optical generation of excitons in the donor phase, exciton diffusion to the interface and exciton dissociation leading to the creation of charge transfer excitons at the donor-acceptor interface. The probability $p_{\text {diss }}$ for dissociation of the charge transfer exciton is given by ${ }^{23}$

$$
p_{\mathrm{diss}}=\frac{k_{\mathrm{rec}} n_{\mathrm{i}}^{2}}{k_{\mathrm{rec}} n_{\mathrm{i}}^{2}+k_{\mathrm{f}} \xi_{0}}=\frac{1}{1+\frac{k_{\mathrm{f}} \xi_{0}}{k_{\mathrm{rec}} n_{\mathrm{i}}^{2}}},
$$

where $\xi_{0}$ is the equilibrium concentration of charge transfer excitons and $n_{\mathrm{i}}^{2}=n_{0} p_{0}$ is the product of the equilibrium concentrations $n_{0}$ and $p_{0}$ of electrons in the acceptor material and holes in the donor material. Note that Eq. (1) is often $^{6-11,14}$ written in the form $p_{\text {diss }}=k_{\mathrm{d}} /\left(k_{\mathrm{d}}+k_{\mathrm{f}}\right)$, where the dissociation constant $k_{\mathrm{d}}$ relates to $k_{\text {rec }}$ and $\xi_{0}$ via $k_{\mathrm{d}}$ $=k_{\text {rec }} n_{\mathrm{i}}^{2} / \xi_{0}$. However, using the form of Eq. (1), it becomes 
(a)
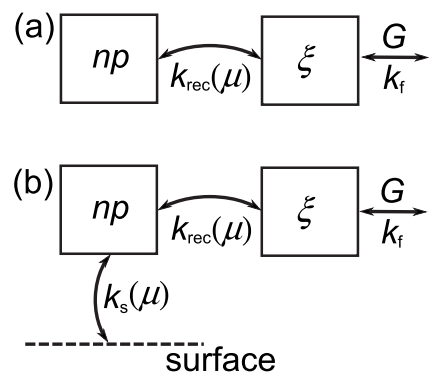

(c)

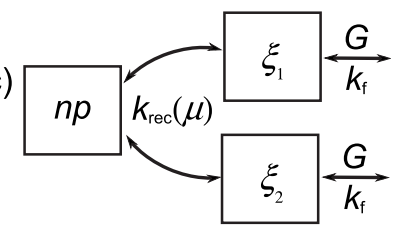

FIG. 1. Scheme of the three discussed device configurations. In the simplest configuration (a), charge transfer excitons $\xi$ are created with the generation rate $G$ and decay with the recombination constant $k_{\mathrm{f}}$. The coupling constant $k_{\mathrm{rec}}(\mu)$ describes the interaction of charge transfer excitons with electrons and holes with concentrations $n$ and $p$, respectively. In case (b), the recombination of free charge carriers at the two interfaces of the absorber with the contact is introduced as a second mobility dependent process. For higher mobilities, it will be more likely that minority carriers recombine at the surfaces. Case (c) represents a simplification of the continuous distribution of binding energy of charge transfer excitons, having only two states with different binding energies and, thus, different equilibrium concentrations $\xi_{0}$.

clear that there is only one constant-namely, $k_{\text {rec}}$-describing the coupling between free carriers and charge transfer excitons, while only the ratio of the equilibrium concentrations can induce asymmetries in the rates of recombination and dissociation.

\section{RESULTS}

\section{A. Zero surface recombination}

In order to investigate whether the solar-cell efficiency may decrease with mobility, we simulate the current/voltage curves of a bulk heterojunction solar cell with the commercial device simulator advanced semiconductor analysis (ASA) as described in Ref. 23 for different values of the electron mobility $\mu_{\mathrm{n}}$. We choose the same parameters as given by Ref. 14 , i.e., an absorber thickness $d=100 \mathrm{~nm}$, an effective band gap $E_{\mathrm{g}}=1.0 \mathrm{eV}$, a constant generation rate $G=6 \times 10^{21} \mathrm{~cm}^{-3} \mathrm{~s}^{-1}$, a spatially averaged relative dielectric constant $\varepsilon_{\mathrm{r}}=3.4$, a ratio between electron and hole mobilities of $\mu_{\mathrm{n}} / \mu_{\mathrm{p}}=10$, an average separation of the electron and hole in the charge transfer state $a=1.8 \mathrm{~nm}$, and a recombination constant $k_{\mathrm{f}}=1.4 \times 10^{4} \mathrm{~s}^{-1}$. The effective densities of states $N_{\mathrm{C}}$ and $N_{\mathrm{V}}$ for conduction and valence band needed for calculation of $n_{\mathrm{i}}^{2}=N_{\mathrm{C}} N_{\mathrm{V}} \exp \left(-E_{\mathrm{g}} / k T\right)$ are taken from Ref. 8 as $N_{\mathrm{C}}=N_{\mathrm{V}}=2.5 \times 10^{19} \mathrm{~cm}^{-3}$. The temperature $T$ is assumed to be $T=300 \mathrm{~K}$ leading to a thermal energy of $k T \approx 26 \mathrm{meV}$. The coupling constant between free carriers and charge transfer excitons is given by a modified Langevin rate as ${ }^{24}$

$$
k_{\mathrm{rec}}=\frac{q \min \left(\mu_{\mathrm{n}}, \mu_{\mathrm{p}}\right)}{\varepsilon_{0} \varepsilon_{\mathrm{r}}} .
$$

To make our simulation consistent with the approach of Ref. 14 , we use ${ }^{7}$

$$
k_{\mathrm{d}}=\frac{k_{\mathrm{rec}} n_{\mathrm{i}}^{2}}{\xi_{0}}=k_{\mathrm{rec}} \frac{3}{4 \pi a^{3}} e^{-E_{\mathrm{B}} / k T}\left[1+b+\frac{b^{2}}{3}+\frac{b^{3}}{18}+\cdots\right],
$$

which defines the equilibrium concentration $\xi_{0} .{ }^{23}$ Here, $E_{\mathrm{B}}(a)=q^{2} / 4 \pi \varepsilon_{0} \varepsilon_{\mathrm{r}} a$ is the binding energy of the charge transfer exciton, $b=q^{3} F / 8 \pi \varepsilon_{0} \varepsilon_{\mathrm{r}}(k T)^{2}, q$ is the elementary charge, and $F$ is the electric field. The smaller the distance $a$, the higher is the Coulomb binding energy $E_{\mathrm{B}}$. A high $E_{\mathrm{B}}$, in turn, leads to a small energy of the charge transfer exciton. In general, smaller energies of an electron hole pair or an exciton automatically lead to higher equilibrium concentrations if the density of states is unchanged. This corresponds to the fact that high band-gap materials have generally lower intrinsic carrier concentrations than low band-gap materials. In our case of the charge transfer exciton, Eq. (3) directly gives the proportionality $\xi_{0} \sim \exp \left(E_{\mathrm{B}} / k T\right)$.

The model used here is chosen such that it is consistent with the approach used in Ref. 14, despite some results in literature that disagree with several aspects of the current model. For instance, the description of recombination via the modified Langevin Eq. (2) was not reproduced by Monte Carlo modeling by Groves et al. ${ }^{25}$ In addition, several authors $^{26-29}$ reported Monte Carlo simulations that showed a behavior different from that predicted by Onsager-Braun theory. Thus, it is necessary to mention the crucial parts of the model that will lead to qualitatively the same results as will be discussed in the following. Most important for our discussion about the mobility dependence to be relevant is that recombination rates increase with mobility. It is not necessary that they increase in exactly the same way (linear with the smaller mobility) as described in Eq. (2). This increase in recombination rate with mobility has also been predicted by Ref. 25. In addition, the exact efficiency of exciton dissociation is also not relevant, it is only important that smaller separation distances of electrons and holes lead to higher Coulomb attraction. Therefore, we conclude that despite the vivid discussion of the exact description of dissociation and recombination processes at the donor-acceptor interface, the qualitative result of our study is quite general.

The squares in Fig. 2 show (a) the open circuit voltage $V_{\mathrm{oc}}$ and (b) the efficiency $\eta$ as a function of electron mobility $\mu_{\mathrm{n}}$ for the configuration given by Fig. 1(a). For this configuration, the only possible recombination path is the creation of a charge transfer exciton and decay with the constant $k_{\mathrm{f}}$. Minority carrier recombination at the contacts is explicitly prohibited, i.e., the surface recombination velocity $S_{\mathrm{C}}$ for minority carriers at both contacts is $S_{\mathrm{C}}=0$. Figure 2(a) shows that the open circuit voltage $V_{\mathrm{oc}}$ is constant in this situation and Fig. 2(b) shows that the efficiency $\eta$ increases monotonously and asymptotically approaches a maximum for $\mu_{\mathrm{n}}$ $>10^{-1} \mathrm{~cm}^{2} /(\mathrm{Vs})$. Thus, the mobility dependent parameter $k_{\text {rec }}$ cannot lead to a decrease in open circuit voltage in this 


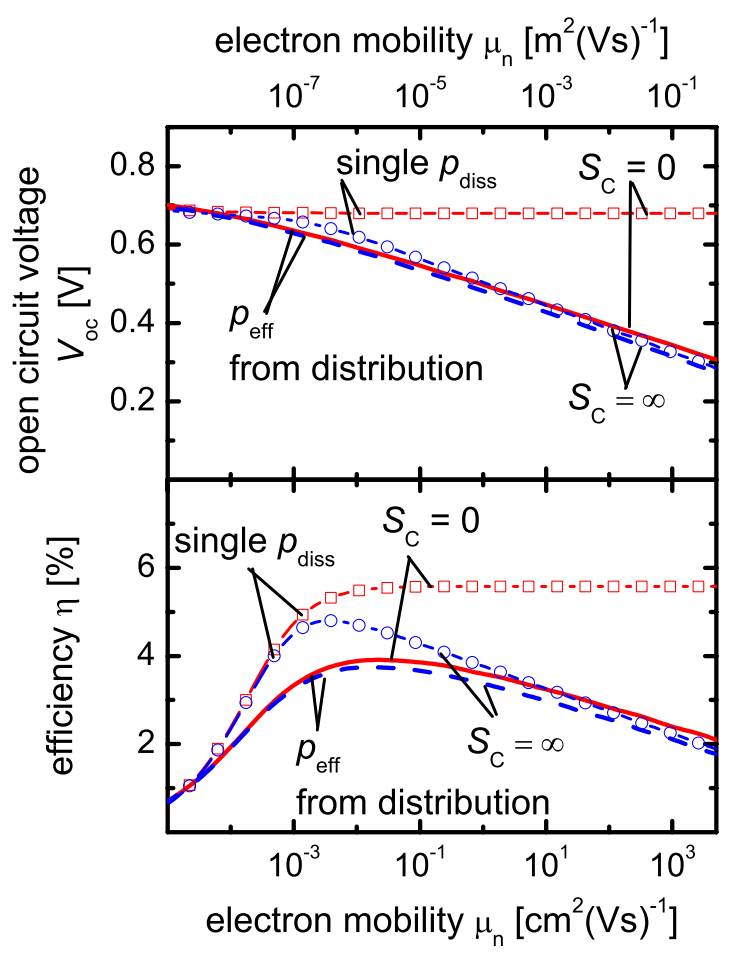

FIG. 2. (Color online) (a) Open circuit voltage and (b) efficiency as a function of electron mobility for four configurations: no surface recombination $\left(S_{\mathrm{C}}=0\right)$ with a single charge transfer state [cf. Fig. 1 (a) ] and thus using Eq. (1) for $p_{\text {diss }}$ (open squares), infinite surface recombination $\left(S_{C}=\infty\right)$ with a single charge transfer state [cf. Fig. $1(\mathrm{~b})$, open circles], no surface recombination $\left(S_{\mathrm{C}}=0\right)$ with a distribution of charge transfer states using Eq. (5) to calculate $p_{\text {eff }}$ (solid line), and infinite surface recombination $\left(S_{\mathrm{C}}=\infty\right)$ combined with a distribution of charge transfer states (dashed line). Except for the first case [open squares, cf. Fig. 1(a)], all other configurations lead to a similar decay of open circuit voltage with mobility, leading to a finite optimum mobility for the efficiency.

simple configuration. This is due to the fact that in open circuit, the net current between charge transfer excitons and free carriers becomes zero and, thus, the chemical potential of charge transfer excitons equals the quasi-Fermi-level splitting of free carriers $\left(\xi / \xi_{0}=n p / n_{\mathrm{i}}^{2}\right)$ independent of the value of $k_{\mathrm{rec}}$. The recombination rate $k_{\mathrm{f}} \xi=k_{\mathrm{f}} \xi_{0} \times n p / n_{\mathrm{i}}^{2}$ will then also be independent of $k_{\text {recc }}$. This result also implies that the argument of Mandoc et al. ${ }^{14}$ that high mobilities will lead to a low quasi-Fermi-level splitting and thus to a low open circuit voltage is not correct if the surface recombination velocity for minorities is zero. Although high mobilities lead to a low quasi-Fermi-level splitting under short circuit conditions, this is not necessarily the case in open circuit. Under applied bias, carriers are also injected from the contacts and thus high mobilities only lead to a homogenization of Fermi levels within the device.

\section{B. Infinite surface recombination}

Figure 1(b) shows a configuration where the surface at both sides of the active region of the device has a finite surface recombination velocity $S_{\mathrm{C}}$. In this case, the mobility influences not only the coupling $k_{\text {rec }}$ between free carriers and charge transfer excitons, as well as the movement of charge carriers to their respective contacts, but also the transport of minorities to the opposite contact, where the minorities may recombine. The circles in Fig. 2 show the open circuit voltage $V_{\mathrm{oc}}$ and the efficiency $\eta$ for the case of infinite surface recombination $S_{\mathrm{C}} \rightarrow \infty$. Now, the open circuit voltage decreases strongly with increasing mobility and the efficiency has a peak at rather low mobilities $\mu_{\mathrm{n}}$ $<10^{-2} \mathrm{~cm}^{2} /(\mathrm{Vs})$. The increase in mobility makes the new recombination pathway via the surfaces more effective and thus leads to a decrease in efficiency once the mobility is sufficient to guarantee an efficient extraction of carriers. This result is therefore in clear agreement with Street, ${ }^{30}$ who concluded that "low mobility might help mitigate a particular loss mechanism in a certain material..."

The identification of surface recombination as a possible loss mechanism, especially for higher mobility organic solar cells, leads to the question of the typical magnitude of surface recombination velocities in organic solar cells. From the fact that organic solar cells always feature a crossover point between dark and illuminated current/voltage curves, where the photocurrent changes its direction, we conclude that very low surface recombination velocities for minorities are not possible. Otherwise, electron extraction at the hole contact and hole extraction at the electron contact would not be working efficiently and would hinder the sign reversal in photocurrent. However, not only very low surface recombination efficiencies but also very high ones are not possible. An upper limit to $S_{\mathrm{C}}$ is given by the requirement that $S_{\mathrm{C}}$ $=v_{\mathrm{th}} \sigma_{\mathrm{d}} N_{\mathrm{d}}$ (Ref. 31) can never exceed the thermal velocity $v_{\text {th }}$, since the product $\sigma_{\mathrm{d}} N_{\mathrm{d}}$ of capture cross-section $\sigma_{\mathrm{d}}$ and defect density $N_{\mathrm{d}}$ at the surface cannot exceed unity. In case $\sigma_{\mathrm{d}} N_{\mathrm{d}}=1$, the surface is perfectly defective, meaning that every minority carrier, diffusing to the surface, recombines immediately. The surface recombination velocity $S_{\mathrm{C}}$ is then only limited by transport to the surface, i.e., by $v_{\text {th }}$. From typical values ${ }^{32}$ used for Monte Carlo simulations, we estimate $S_{\mathrm{C}}=7 \times 10^{5} \mathrm{~cm} / \mathrm{s}$ as an upper limit for $S_{\mathrm{C}}$ for typical mobilities. Even if current surface recombination velocities may be closer to infinity than to zero, it is certainly possible to decrease surface recombination currents by using band offsets for the respective minorities at the contacts. This concept has been known for a long time in photovoltaics ${ }^{33}$ and has been applied in both inorganic ${ }^{34,35}$ and organic devices. ${ }^{36}$

\section{Charge transfer state distribution}

In the model of Refs. 7 and 8, the dissociation probability $p_{\text {diss }}$ from Eq. (1) is not directly used, but is first integrated with a normalization function to account for a distribution

$$
d F(a)=\frac{4}{\sqrt{\pi} \bar{a}^{3}} a^{2} e^{-a^{2} / \bar{a}^{2}} d a
$$

of separation distances $a$ of the bound electron/hole pair, where $\bar{a}$ denotes the average separation distance. Then, the dissociation probability $p_{\text {diss }}$ is replaced by the effective or average dissociation probability 


$$
p_{\text {eff }}=\frac{4}{\sqrt{\pi} \bar{a}^{3}} \int_{0}^{\infty} p_{\text {diss }}(a) a^{2} e^{-a^{2} / \bar{a}^{2}} d a .
$$

In the simplest schematic representation of this integral [depicted in Fig. 1(c)], the generation of free charge carriers happens via more than one (e.g., two) charge transfer state with different separation distance $a$. Then, the different charge transfer states (say, numbered by index $i$ ) must have different equilibrium concentration $\xi_{0 \mathrm{i}}$, since the equilibrium concentration depends on the separation distance $a$. This is because a high separation distance $a$ leads to a low Coulomb binding energy $E_{\mathrm{B}}(a)=q^{2} / 4 \pi \varepsilon_{0} \varepsilon_{\mathrm{r}} a$, meaning that the equilibrium concentration of such a state is rather low. If instead the separation distance is very small, the carriers feel attractive forces, making the state energetically more favorable, which implies that the equilibrium concentration $\xi_{0 \mathrm{i}}$ is higher. Thus, the assumption that it is physically reasonable to consider a distribution of separation distances of the charge transfer excitons implies that also the equilibrium concentrations are distributed. In the following, we therefore explore the implications of distributed equilibrium concentrations.

In order to investigate the currents flowing between the distribution of charge transfer states and the free carriers, we need to define the relation between the dissociation probability $p_{\text {diss }}$ or effective dissociation probability $p_{\text {eff }}$ and the recombination constant $k_{\text {rec }}$ with the current. The starting point for the derivation of the current is the steady-state solution for the occupation of a charge transfer state with distance $a$ under a photogeneration rate $G$, which is given by

$$
-k_{\mathrm{f}} \xi_{\mathrm{i}}+k_{\mathrm{f}} \xi_{0 \mathrm{i}}(a)-k_{\mathrm{rec}} n_{\mathrm{i}}^{2} \frac{\xi_{\mathrm{i}}}{\xi_{0 \mathrm{i}}(a)}+k_{\mathrm{rec}} n p-G=0 .
$$

Resolving Eq. (6) for $\xi_{\mathrm{i}}$ and using the result to calculate the current density $j_{\mathrm{i}}$ carried by the transfer state yields

$$
\begin{aligned}
j_{\mathrm{i}}(a) & =q k_{\mathrm{rec}} \frac{\left(n p-n_{\mathrm{i}}^{2}\right) k_{\mathrm{f}} \xi_{0 \mathrm{i}}(a)-G n_{\mathrm{i}}^{2}}{k_{\mathrm{f}} \xi_{0 \mathrm{i}}(a)+k_{\mathrm{rec}} n_{\mathrm{i}}^{2}} \\
& =q k_{\mathrm{rec}}\left(n p-n_{\mathrm{i}}^{2}\right)\left[1-p_{\mathrm{diss}}(a)\right]-q p_{\mathrm{diss}}(a) G,
\end{aligned}
$$

where we have used Eq. (1) and $q$ denotes the elementary charge. Integration of Eq. (7) over $d F(a)$ finally yields the current density of a distribution of transfer states

$$
\begin{aligned}
j_{\mathrm{F}}(\bar{a}) & =\int\left\{q k_{\mathrm{rec}}\left(n p-n_{\mathrm{i}}^{2}\right)\left[1-p_{\mathrm{diss}}(a)\right]-q p_{\mathrm{diss}}(a) G\right\} d F(a) \\
& =q k_{\mathrm{rec}}\left(n p-n_{\mathrm{i}}^{2}\right)\left[1-p_{\mathrm{eff}}(\bar{a})\right]-q p_{\mathrm{eff}}(\bar{a}) G
\end{aligned}
$$

The solid and the dashed lines in Fig. 2 show the simulation of open circuit voltage $V_{\mathrm{oc}}$ and efficiency $\eta$ using Eq. (8) for the local current density for the case of both zero and infinite surface recombinations $S_{\mathrm{C}}$. In contrast to the simulations using Eq. (1), the open circuit voltage decays with increasing mobility even for $S_{\mathrm{C}}=0$.

In order to understand the mobility dependence of $V_{\mathrm{oc}}$ when using Eq. (5), we first consider the simplified scheme of only two types of charge transfer states as sketched in Fig. 3 for (a) low and (b) high coupling $k_{\text {rec }}(\mu)$. Note that as (a) weak coupling, low $k_{\text {rec }}$, low $\mu$ $E \uparrow$

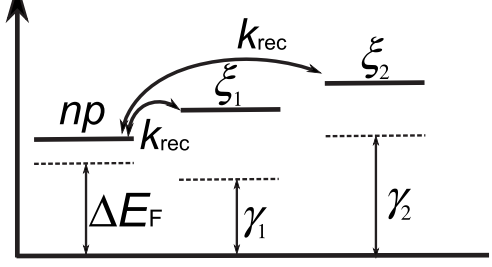

(b) strong coupling, high $k_{\text {rec }}$, high $\mu$ E个

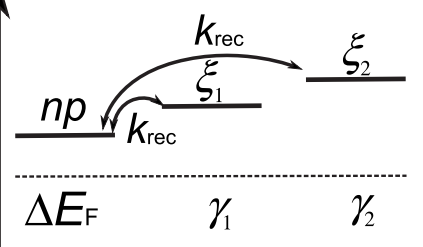

FIG. 3. Explanation of the mobility dependence of the open circuit voltage of a distribution of charge transfer states using a simplified scheme with two charge transfer states with different binding energies and thus with different equilibrium concentrations $\xi_{01}$ and $\xi_{02}\left(\xi_{01}>\xi_{02}\right)$. In the case of weak coupling, corresponding to a low $k_{\text {rec }}$ and a low mobility $\mu$ (cf. Fig. 2), the chemical potentials of the two charge transfer states are different and the Fermilevel splitting of the free carriers is in between according to Eq. (6). In the case of higher mobilities and more efficient coupling between free carriers and charge transfer states, the chemical potentials $\gamma_{1}$ and $\gamma_{2}$ approach each other and the quasi-Fermi-level splitting $\Delta E_{\mathrm{F}}$. One of the two states has a higher binding energy and thus a higher equilibrium concentration leading to a lower chemical potential for given values of $G$ and $k_{\mathrm{f}}$. In case of the strong coupling, the quasiFermi-level splitting of the free carriers (and thus the open circuit voltage) are limited by the lower chemical potential of the charge transfer state with the high equilibrium concentration

shown in Fig. 1(c), there is no direct coupling between the two charge transfer states. Interaction between the two states is only possible via the free carriers. This corresponds to the situation where the charge transfer states are spatially separated from each other. Note that the spatial separation of the charge transfer states is also implicitly assumed in the integral given by Eq. (5).

According to Eq. (2) the coupling between the charge transfer states and the free charge carriers depends on the mobility. Low coupling requires low mobilities, while a high coupling corresponds to a high mobility (cf. Fig. 2). In the limit of zero coupling, the two charge transfer states will have a chemical potential $\gamma_{\mathrm{i}}=k T \ln \left(\xi_{\mathrm{i}} / \xi_{0 \mathrm{i}}\right)$ that only depends on the generation rate $G$ and the respective recombination rate $R_{\mathrm{i}}=k_{\mathrm{f}} \xi=k_{\mathrm{f}} \xi_{0 \mathrm{i}} \exp \left(\gamma_{\mathrm{i}} / k T\right)$, i.e., the chemical potential is independent of the concentration of free charge carriers and, consequently, also independent from the other charge transfer state(s). The chemical potential of the exciton in state $i$ for zero coupling to free carriers and other charge transfer excitons follows from the open circuit condition $G=R$ as:

$$
\gamma_{\mathrm{i}}=k T \ln \left(\frac{G}{k_{\mathrm{f}} \xi_{0 \mathrm{i}}}\right) \text {. }
$$

Thus, the state with the high equilibrium concentration (corresponding to a low energy) has the smaller chemical poten- 
tial. This result corresponds to the fact that solar cells made from low band-gap semiconductors have usually higher dark currents and lower open circuit voltages than devices made from high band-gap semiconductors.

If the coupling constant is still low but positive, as shown in Fig. 3(a), the difference in the chemical potentials between the charge transfer excitons is still there and the quantity of interest, namely, the quasi-Fermi-level splitting $\Delta E_{\mathrm{F}}$ of the free carriers, depends on both $\gamma_{1}$ and $\gamma_{2}$. Due to the requirement of zero net current under open circuit conditions, follows:

$$
k_{\mathrm{rec}}\left[\frac{\xi_{1}}{\xi_{01}}+\frac{\xi_{2}}{\xi_{02}}-2 \frac{n p}{n_{\mathrm{i}}^{2}}\right]=0 .
$$

Thus, the quasi-Fermi-level splitting $\Delta E_{\mathrm{F}}=k T \ln \left(n p / n_{\mathrm{i}}^{2}\right)$ of the free carriers will be in between the chemical potentials of the two charge transfer states.

As shown in Fig. 3(b), the quasi-Fermi-level splitting of free carriers and the chemical potential of the charge transfer excitons are the same $\left(\xi_{1} / \xi_{01}=\xi_{2} / \xi_{02}=n p / n_{\mathrm{i}}^{2}\right)$ if the coupling is very efficient $\left(k_{\text {rec }}\right.$ is high). The recombination $k_{\mathrm{f}} \xi_{01} \exp \left(\gamma_{1} / k T\right)$ via the charge transfer state with the lower band gap is enhanced and its chemical potential $\gamma_{1}$ is increased compared to Fig. 3(a). This causes also the whole system to have more recombination and the concentration $n p / n_{\mathrm{i}}^{2}$ of free carriers to decrease compared with less efficient coupling. The validity of this claim becomes more evident when solving Eq. (10) for the normalized free carrier concentration $n p / n_{\mathrm{i}}^{2}$. If we consider all rate equations for $\xi_{1} / \xi_{01}$ and $\xi_{2} / \xi_{02}$ as done, e.g., in Ref. 23, we receive under open circuit conditions

$$
\frac{n p}{n_{\mathrm{i}}^{2}}-1=\frac{G\left(k_{\mathrm{f}}\left[\xi_{01}+\xi_{02}\right]+2 k_{\mathrm{rec}} n_{\mathrm{i}}^{2}\right)}{k_{\mathrm{rec}} n_{\mathrm{i}}^{2} k_{\mathrm{f}}\left[\xi_{01}+\xi_{02}\right]+2 k_{\mathrm{f}}^{2} \xi_{01} \xi_{02}},
$$

which simplifies to

$$
\frac{n p}{n_{\mathrm{i}}^{2}}-1=\frac{G}{k_{\mathrm{f}} \xi_{0}}
$$

if the equilibrium concentrations and the distances are the same, i.e., $\xi_{01}=\xi_{02}$. From the derivative of Eq. (11)

$$
\frac{\partial n p / n_{\mathrm{i}}^{2}}{\partial k_{\mathrm{rec}}}=\frac{-G n_{\mathrm{i}}^{2} k_{\mathrm{f}}\left(k_{\mathrm{f}} \xi_{01}-k_{\mathrm{f}} \xi_{02}\right)^{2}}{\left(k_{\mathrm{rec}} n_{\mathrm{i}}^{2} k_{\mathrm{f}}\left[\xi_{01}+\xi_{02}\right]+2 k_{\mathrm{f}}^{2} \xi_{01} \xi_{02}\right)^{2}} \leq 0,
$$

follows directly that the quasi-Fermi-level splitting $\Delta E_{\mathrm{F}}$ $=k T \ln \left(n p / n_{\mathrm{i}}^{2}\right)$ of the free carriers decreases monotonously with increasing $k_{\text {rec }}$ and thus with increasing mobility. Only in the trivial case that the equilibrium concentration is the same for the two charge transfer states $\left(\xi_{01}=\xi_{02}\right)$, the derivative in Eq. (13) vanishes implying a mobility independent open circuit voltage, as is the case for the configuration shown in Fig. 1(a).

Thus, at higher mobilities, the open circuit voltage decreases due to current flowing from charge transfer states with low equilibrium concentration to those with high equilibrium concentration. To some extent, this situation is similar to that obtained in inorganic solar cell absorbers with fluctuating band-gap energy. ${ }^{37}$ Here, the open circuit voltage

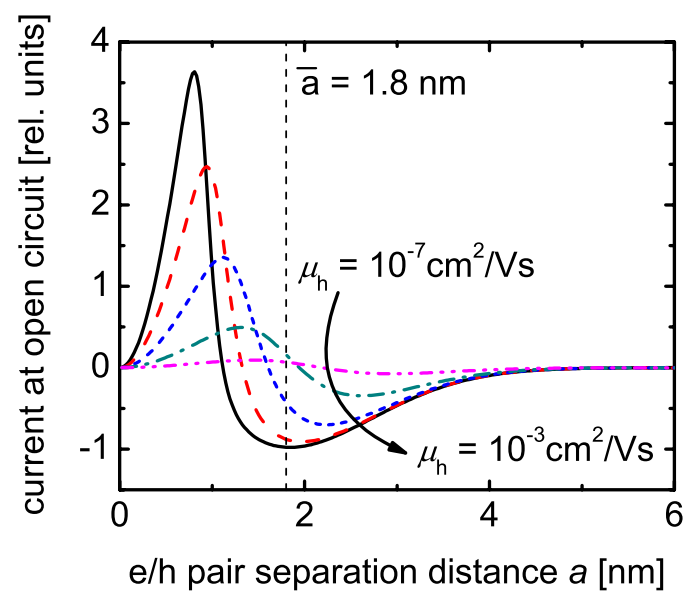

FIG. 4. (Color online) Currents flowing at open circuit from the free carriers to the charge transfer displayed versus $e / h$-pair separation. Due to the open circuit condition only the net current-i.e., the integral over the current-is zero, while exchange currents flow from states with high separation distance $a$ to states with low $a$. For higher mobilities, the better coupling between charge transfer states and free carriers leads to an increase in these exchange currents. In addition, for higher mobilities, the states that receive $e / h$ pairs are shifted to smaller separations $a$, leading to more recombination current.

decreases due to the flow of charge carriers from high bandgap regions toward regions with lower band-gap energies.

Going back from the simple two-state model to the complete distribution defined by Eq. (4), Fig. 4 visualizes the current flow at open circuit between the charge transfer states at different separation distances $a$. The net current, being the integral over the curves is always zero, since the simulated device is at open circuit. For simplicity, the electric field has been set constant to $F=\left(V_{\mathrm{bi}}-V_{\mathrm{oc}}\right) / d$. For low coupling and small mobilities, only small currents flow from the charge transfer states with high separation distance to those with low separation distance $a$. For higher mobilities, however, most charge transfer states with higher distance $a$ inject $e / h$ pairs into few states with very low $a$ where they recombine. Thus, the higher the mobility, the more detrimental recombination via charge transfer states with small separation distance becomes.

\section{CONCLUSIONS}

The present paper has demonstrated two ways how the counterintuitive result, that better transport properties, i.e., higher charge-carrier mobilities, in a bulk heterojunction solar cell can lead to a reduction in the device performance, i.e., reduced efficiency. Two assumptions made in Refs. 14 and 15 eventually lead to the prediction of an optimum device performance at finite mobilities. Both features have in common that there is more than one generationrecombination path in the device that is affected by mobility.

The first feature, the assumption of infinite or very high surface recombination, represents an additional, parasitic recombination channel. At high carrier mobilities, this channel increasingly dominates recombination whereas at the same 
time high mobilities are required for carrier collection. Thus, an optimum mobility is determined by the balance between interface recombination (defining the open circuit voltage) and carrier collection (defining the short circuit current density).

The second feature involves a distribution of separation distances of the charge transfer exciton. Interestingly, this scenario does not involve a parasitic recombination path. In contrast, all charge transfer states involve recombination and generation of free charge carriers and are therefore photovoltaically useful. However, the low energy of the states with short separation distance implies that these states dominate the recombination in a way that leads to a reduction in efficiency in the limit of high mobilities.

The practical consequences of the present results are twofold: (i) a reduction in contact recombination in bulk heterojunction solar cells is crucial. This task represents a more technical problem that is furthermore not specific to organic solar cells. (ii) In contrast, Langevin recombination would represent a fundamental limitation for the efficiency of organic bulk heterojunction solar cells. Although disorder in the distribution of energetic states is certainly reasonable to assume, the suitable theoretical description of recombination via a distribution of charge transfer states should be further discussed. This is particularly important for the distribution functions used in current models; especially, since these were originally developed for quite different systems. ${ }^{38,39}$

\section{ACKNOWLEDGMENTS}

The authors would like to thank C. Deibel (University of Würzburg) for fruitful discussions and U. Aeberhard (FZ Jülich) for carefully reading the manuscript.
${ }^{1}$ N. S. Sariciftci, L. Smilowitz, A. J. Heeger, and F. Wudl, Science 258, 1474 (1992).

${ }^{2}$ G. Yu, J. Gao, J. C. Hummelen, F. Wudl, and A. J. Heeger, Science 270, 1789 (1995).

${ }^{3}$ H. Hoppe and N. S. Sariciftci, J. Mater. Res. 19, 1924 (2004).

${ }^{4}$ P. W. M. Blom, V. D. Mihailetchi, L. J. A. Koster, and D. E. Markov, Adv. Mater. 19, 1551 (2007).

${ }^{5}$ S. R. Forrest, MRS Bull. 30, 28 (2005).

${ }^{6}$ C. L. Braun, J. Chem. Phys. 80, 4157 (1984).

${ }^{7}$ V. D. Mihailetchi, L. J. A. Koster, J. C. Hummelen, and P. W. M. Blom, Phys. Rev. Lett. 93, 216601 (2004).

${ }^{8}$ L. J. A. Koster, E. C. P. Smits, V. D. Mihailetchi, and P. W. M. Blom, Phys. Rev. B 72, 085205 (2005).

${ }^{9}$ D. W. Sievers, V. Shrotriya, and Y. Yang, J. Appl. Phys. 100, 114509 (2006).

${ }^{10}$ H. H. P. Gommans, M. Kemerink, J. M. Kramer, and R. A. J. Janssen, Appl. Phys. Lett. 87, 122104 (2005).

${ }^{11}$ J. D. Kotlarski, P. W. M. Blom, L. J. A. Koster, M. Lenes, and L. H. Slooff, J. Appl. Phys. 103, 084502 (2008).

${ }^{12}$ D. Veldman, Ö. Ipek, S. C. J. Meskers, J. Sweelssen, M. M. Koetse, S. C. Veenstra, J. M. Kroon, S. S. van Bavel, J. Loos, and R. A. J. Janssen, J. Am. Chem. Soc. 130, 7721 (2008).

${ }^{13}$ J. J. Benson-Smith, L. Goris, K. Vandewal, K. Haenen, J. V. Manca, D. Vanderzande, D. D. C. Bradley, and J. Nelson, Adv. Funct. Mater. 17, 451 (2007).

${ }^{14}$ M. M. Mandoc, L. J. A. Koster, and P. W. M. Blom, Appl. Phys. Lett. 90, 133504 (2007).

${ }^{15}$ C. Deibel, A. Wagenpfahl, and V. Dyakonov, Phys. Status Solidi (RRL) 2, 175 (2008).

${ }^{16}$ J. Mattheis, J. H. Werner, and U. Rau, Phys. Rev. B 77, 085203 (2008).

${ }^{17}$ T. E. Goliber and J. H. Perlstein, J. Chem. Phys. 80, 4162 (1984).

${ }^{18}$ L. Onsager, J. Chem. Phys. 2, 599 (1934).

${ }^{19}$ P. W. Bridgman, Phys. Rev. 31, 101 (1928).
${ }^{20}$ J. Nelson, J. Kirkpatrick, and P. Ravirajan, Phys. Rev. B 69, 035337 (2004).

${ }^{21}$ T. Kirchartz and U. Rau, Phys. Status Solidi A 205, 2737 (2008).

${ }^{22}$ T. Kirchartz, J. Mattheis, and U. Rau, Phys. Rev. B 78, 235320 (2008).

${ }^{23}$ T. Kirchartz, B. E. Pieters, K. Taretto, and U. Rau, J. Appl. Phys. 104, 094513 (2008).

${ }^{24}$ L. J. A. Koster, V. D. Mihailetchi, and P. W. M. Blom, Appl. Phys. Lett. 88, 052104 (2006).

${ }^{25}$ C. Groves and N. C. Greenham, Phys. Rev. B 78, 155205 (2008).

${ }^{26}$ T. Offermans, S. C. J. Meskers, and R. A. J. Janssen, Chem. Phys. 308, 125 (2005).

${ }^{27}$ P. Peumans and S. R. Forrest, Chem. Phys. Lett. 398, 27 (2004).

${ }^{28}$ S. Barth, D. Hertel, Y.-H. Tak, H. Bässler, and H. H. Hörhold, Chem. Phys. Lett. 274, 165 (1997).

${ }^{29}$ C. Groves, R. A. Marsh, and N. C. Greenham, J. Chem. Phys. 129, 114903 (2008)

${ }^{30}$ R. A. Street, Appl. Phys. Lett. 93, 133308 (2008).

${ }^{31}$ S. M. Sze, Physics of Semiconductor Devices (Wiley, New York, 1981), p. 57.

${ }^{32}$ R. A. Marsh, C. Groves, and N. C. Greenham, J. Appl. Phys. 101, 083509 (2007).

${ }^{33}$ E. Yablonovitch, T. Gmitter, R. M. Swanson, and Y. H. Kwark, Appl. Phys. Lett. 47, 1211 (1985).

${ }^{34}$ M. Taguchi, A. Terakawa, E. Maruyama, and M. Tanaka, Prog. Photovoltaics 13, 481 (2005).

${ }^{35}$ P. J. Rostan, U. Rau, V. X. Nguyen, T. Kirchartz, M. B. Schubert, and J. H. Werner, Sol. Energy Mater. Sol. Cells 90, 1345 (2006).

${ }^{36}$ P. E. Keivanidis, S.-H. Khong, P. K. H. Ho, N. C. Greenham, and R. H. Friend, Appl. Phys. Lett. 94, 173303 (2009).

${ }^{37}$ U. Rau and J. H. Werner, Appl. Phys. Lett. 84, 3735 (2004).

${ }^{38}$ A. Mozumder, J. Chem. Phys. 60, 4305 (1974).

${ }^{39}$ D. S. Sethi, H. T. Choi, and C. L. Braun, Chem. Phys. Lett. 74 223 (1980). 